\title{
The relationship between tumour budding, the tumour microenvironment and survival in patients with invasive ductal breast cancer
}

\author{
F J A Gujam*,1,2, D C McMillan ${ }^{1}$, Z M A Mohammed ${ }^{3}$, J Edwards ${ }^{2}$ and J J Going ${ }^{4}$ \\ ${ }^{1}$ Academic Unit of Surgery, College of Medical, Veterinary and Life Sciences-University of Glasgow, Royal Infirmary, Glasgow, UK; \\ ${ }^{2}$ Unit of Experimental Theraputics, Institute of Cancer Sciences, College of Medical, Veterinary and Life Sciences-University of \\ Glasgow, Glasgow, UK; ${ }^{3}$ University Departments of Pathology, Faculty of Veterinary Medicine, Omar Almukhtar University, Al \\ bayda, Libya and ${ }^{4}$ University Section of Pathology, College of Medical, Veterinary and Life Sciences-University of Glasgow, \\ Southern General Hospital, Glasgow, UK
}

Background: Tumour budding has previously been reported to predict survival in several solid organ tumours, including breast; however, whether this is independent of other aspects of the tumour microenvironment is unknown. In the present study, the relationship between tumour budding, the tumour microenvironment and survival was examined in patients with invasive ductal breast cancer.

Methods: Patients presenting between 1995 and 1998 were studied $(n=474)$. Using routine pathological sections, tumour budding was measured at the invasive margin and its association with clinicopathological characteristics and cancer-specific survival (CSS) was examined.

Results: Tumour budding was associated with several adverse pathological characteristics, including lymph node involvement, lymph vessel invasion (LVI), increased tumour stroma percentage (TSP) and weaker local inflammatory infiltrative. Tumour budding was associated with reduced CSS (hazard ratio (HR) 2.08, 95\% confidence interval (CI) 1.14-3.09, $P=0.004$ ), independent of nodal status, molecular subtypes, tumour necrosis, CD8 + , CD138 +, LVI, blood vessel invasion and TSP. Further, tumour budding was independently associated with reduced CSS in node-negative patients (HR 2.63,95\% Cl 1.16-5.92, $P=0.020$ ) and those who have low TSP (HR 1.98, 95\% Cl 1.09-3.57, $P=0.024)$ and high-grade local inflammatory infiltrative ( $\mathrm{HR} 2.27,95 \% \mathrm{Cl} 1.35-5.36, P=0.014)$.

Conclusions: Tumour budding was a significant predictor of survival in patients with invasive ductal breast cancer, independent of adverse pathological characteristics and components of tumour microenvironment. The present study further confirms the clinical utility of both tumour and host-based factors of tumour microenvironment.

In the United Kingdom, >49000 women are diagnosed with breast cancer in 2011 and approximately $80 \%$ survive at least 5 years (Cancerstats, 2014). Breast cancer is a heterogeneous disease and a number of different molecular subtypes have emerged; however, standard histopathological characteristics remain the most useful prognostic factors. It is clear for such heterogeneous disease that the need to effectively stratify patients according to likely outcome remains important. This should be done against a comprehensive characterisation of the tumour and its microenvironment. For example, there is now increased recognition of the importance of the tumour microenvironment, including tumour necrosis, host local inflammatory responses and tumour stroma, in cancer progression and survival (Richards et al, 2011; Mohammed et al, 2012a; Freeman et al, 2013).

Recently, the tumour budding, which refers to detachment of single or cluster of up to five cancer cells scattered in stroma at the invasive front of tumour (Ueno et al, 2002; Prall et al, 2005; Lugli et al, 2009), has been proposed as an important determinant of

*Correspondence: Dr FJA Gujam; E-mail: f.gujam.1@research.gla.ac.uk

Received 13 May 2015; revised 8 July 2015; accepted 14 July 2015; published online 11 August 2015

(c) 2015 Cancer Research UK. All rights reserved 0007-0920/15 
progression and survival in a number of solid cancers (Hase et al, 1993; Ueno et al, 2002; Prall et al, 2005; Choi et al, 2007; Koike et al, 2008; Masugi et al, 2010; Koyuncuoglu et al, 2012; Taira et al, 2012). In particular, tumour budding is thought to be an early step in cancer metastasis as budded cells have the characteristics of epithelial-mesenchymal transition (EMT) (Masugi et al, 2010; Zlobec and Lugli, 2010; Koyuncuoglu et al, 2012; Lugli et al, 2012; Taira et al, 2012; Liang et al, 2013; Dawson and Lugli, 2015), which is a crucial step during carcinoma progression and metastasis (Kalluri and Weinberg, 2009).

In breast cancer, there is still limited information about the role of tumour budding in breast cancer (Liang et al, 2013; Salhia et al, 2015). Liang et al (2013) has reported the significance of budding in small breast cancer cohort $(n=160)$ with limited follow-up and only reported budding effect on overall survival but did not report on cancer-specific survival (CSS) as an end point. The second report examined the association of tumour budding and clinicopathological characteristics. However, survival analysis was not reported.

Moreover, it is not clear whether the effect of an increased tumour budding on survival is independent of host inflammatory response and other components of the tumour microenvironment. Therefore, the present study aims to examine the relationship between tumour budding, the tumour microenvironment and survival in patients with invasive ductal breast cancer.

\section{MATERIALS AND METHODS}

Patients presenting with invasive ductal breast cancer at Glasgow Royal Infirmary, Western Infirmary and Stobhill Hospital, at West of Scotland, between 1995 and 1998 and had formalin-fixed paraffin-embedded blocks of the primary tumour available for evaluation were studied $(n=474)$. The study was approved by the Research Ethics Committee of the West Glasgow University Hospitals NHS Trust (REC reference is 07/s0704/61).

Clinicopathological data that included age, tumour size, tumour grade, lymph node status, type of surgery and use of adjuvant treatment (chemotherapy, hormonal therapy and/or radiotherapy) were retrieved from the routine reports. Tumour grade was assigned according to Nottingham Grading System. ER and PR status were assessed on tissue microarrays (TMAs) using immunohistochemestry (IHC) with Dako (Glostrup, Denmark) ER antibody and Leica (Wetzlar, Germany) PR antibody and scored according to the American Society of Clinical Oncology and College of American Pathologists guidelines with a cutoff value of $1 \%$ positive tumour nuclei (Hammond et al, 2010). Her2 status were assessed visually using TMAs as previously described, that is, a score $3+$ is regarded as positive; $2+$ is regarded as equivocal, leading to referral for Her-2 FISH; and 0 and $1+$ are regarded as negative (Mohammed et al, 2012b).

Full-section haematoxylin and eosin (H\&E) slides for the 474 patients were used to score local inflammatory infiltrate according to Klintrup criteria. Klintrup scoring of slides was carried out as previously described. Briefly, tumours were scored on four-point scores based on appearances at the tumour invasive margin. A score of 0 signified that there were no inflammatory cells at tumour's invasive margin; score 1 indicated a mild and patchy inflammatory cells; score 2 denoted a prominent band-like inflammatory reaction at the invasive margin; and score 3 revealed a florid cup-like inflammatory infiltrate at the invasive edge (Klintrup et al, 2005; Mohammed et al, 2012a). Individual immune cell types were assessed using IHC staining on TMA sections for macrophages, helper and cytotoxic T-lymphocytes and plasma cells using CD68, CD4, CD8 and CD138 antibodies, respectively (Mohammed et al, 2013).
Full-section H\&E slides were also used to score the tumour stroma percentage (TSP) as previously reported (Gujam et al, $2014 b$ ). Briefly, at $\times 5$ magnification, an area representative of the tumour invasive margin was selected, and then a single field of $\times 10$ magnification was examined, ensuring that tumour cells were present at all four sides of the image and the area of stroma was calculated as a percentage.

Lymph and blood vessel invasion (LVI and BVI, respectively) were assessed, on 2.5- $\mu \mathrm{m}$ thick sections, using IHC staining with the lymphatic endothelial marker D2-40 (Covance, Monoclonal Antibody, SIG-3730, Princeton, NJ, USA) diluted 1:100 and vascular endothelial marker Factor VIII (Mouse Monoclonal Antibody, NCL-L-Vwf, Leica, Newcastle, UK) diluted 1:100 as previously described (Gujam et al, 2014a).

The molecular subtypes were defined as follows: Luminal A: oestrogen (ER) and/or progesterone receptor (PR) positive, Her-2 negative, low proliferative index $(\leqslant 15 \%)$; Luminal B: hormone receptor positive, Her-2 positive, high proliferative index ( $>15 \%)$; Her-2 subtype: Her-2 positive and hormone receptor negative, any proliferative index; and triple negative: Her-2 negative, hormone receptor negative, any proliferative index.

Assessment of tumour budding. Full H\&E-stained sections were used to assess tumour budding at the deepest tumour invasion margin as previously described (Ueno et al, 2002). Tumour sections were scanned using a Hamamatsu NanoZoomer (Welwyn Garden City, Hertfordshire, UK) at $\times 20$ magnification, and visualisation was carried out using the Slidepath Digital Image Hub, version 4.0.1 (Slidepath, Leica Biosystems, Milton Keynes, UK). At $\times 5$ magnification, an area representative of the tumour invasive margin was selected. A grid of $0.385 \mathrm{~mm}^{2}$ size at the five highest budding areas was drawn. Using a $\times 20$ magnification, a tumour budding was counted. A bud was identified as an isolated single cancer cell or a group of up to five cancer cells (Ueno et al, 2002; Prall et al, 2005; Lugli et al, 2009; Figure 1). The highest bud count per field was used as the number of buds. Areas of necrosis or mucin were excluded from the field. To ensure reliability, co-scoring of 60 randomly selected cases was carried out by FJG and the consultant pathologist JJG. The interobserver intraclass

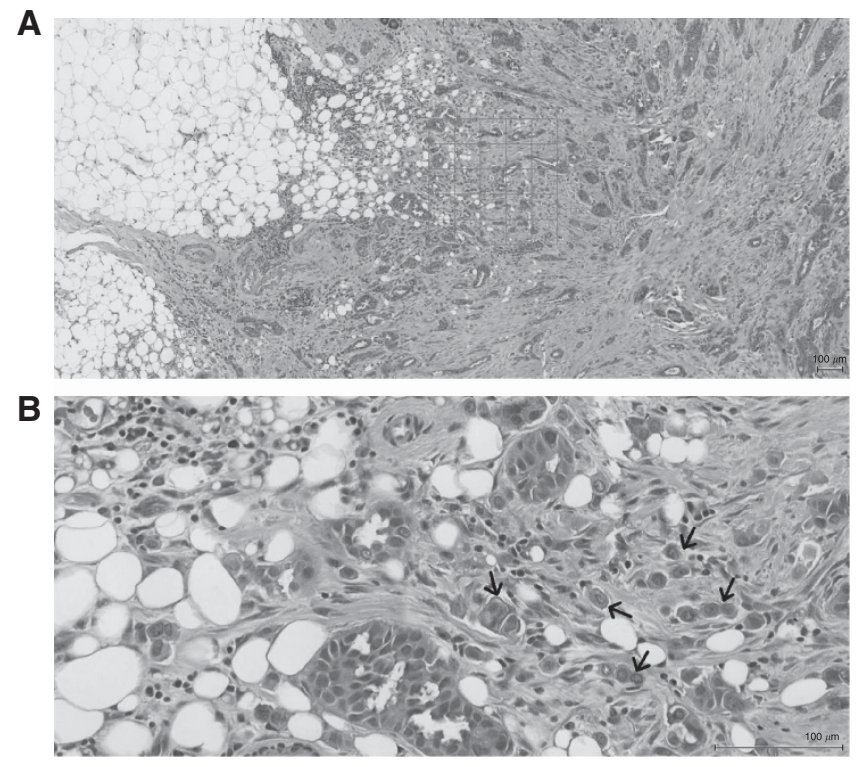

Figure 1. Haematoxylin and eosin stained section of invasive ductal breast cancer. (A) Shows a grid of high tumour budding area at the invasive margin, (B) shows single and clusters of tumour budding (arrows). Original magnification $\times 20$, scale $100 \mu \mathrm{m}$. 
correlation coefficient (ICCC) for the raw continuous scores was $0.813(P<0.001)$. All the slides were then scored by FJG.

Patients were routinely followed up following surgery. Date and cause of death was cross-checked with the cancer registration system and the Registrar General (Scotland). Death records were complete until 31 May 2013 and that served as the censor date.

CSS was measured from the date of primary surgery until the date of death from breast cancer.

Statistical analysis. To identify the cutoff value of tumour budding for survival analysis, the highest budding count per five fields were split into tertiles, and survival analysis between each group using Kaplan-Meier log-rank test was performed (Figure 2A) (Choi et al, 2007; Sy et al, 2010). Subsequently, the first and second tertiles (highest tumour budding count $\leqslant 20$ ) were considered as the low budding group and the third tertile (highest tumour budding $>20$ ) was considered as the high budding group. To simplify all further analysis, patients were subsequently grouped into low tumour budding $(\leqslant 20)$ and high tumour budding $(>20)$.

When ROC analysis was carried out with CSS as an end point, the optimal number of tumour buds was between 15 (sensitivity $=0.55$, specificity $=0.70$ ) and 20 buds (sensitivity $=0.63$, specificity $=0.60$ ) per 5 fields. Therefore, the threshold was set at 20 buds. At this threshold, the AUC was $0.625(P<0.001)$. This was consistent with the threshold derived from the plot of the tertiles (see Figure 2A).

Consistency between the observers was analysed using the ICCC value. The relationships between variables were assessed using contingency table analysis with the $\chi^{2}$ test for linear trend. KaplanMeier analysis was used to examine the effect of tumour budding on CSS. Univariate survival analysis was performed using Cox proportional hazards regression. Variables with $P$-value of $<0.1$ were entered into a multivariable model using a backwards conditional method for all patients, node-negative patients and those who have low TSP and high K-M score. All statistical analyses were two-sided and significance defined as $P$-value $<0.05$. All statistical analysis was performed using the SPSS software version 22 (IBM SPSS, Chicago, IL, USA).

\section{RESULTS}

Table 1 summarises clinicopathological characteristics of patients $(n=474)$. The majority of patients $(70 \%)$ were $>50$ years, had small tumour size $\leqslant 20 \mathrm{~mm}(60 \%)$, had grade II and III tumours $(80 \%)$ and negative lymph node (54\%). The majority had ER-positive (69\%) tumours, PR-positive tumours (61\%) and Her2-negative tumours (80\%). In all, 182 (38\%) had lumpectomy and radiotherapy, and 292 (62\%) had mastectomy and radiotherapy. Also, 243 (51\%) patients received endocrine therapy only, 101 (21\%) patients received adjuvant chemotherapy only and $95(20 \%)$ had both.

A high tumour budding was identified in 167 (35\%) patients. From the distribution chart of the frequency of the buds/5 fields per patient, the number of patients around the cutoff $18,19,20,21$ and 22 buds was 15, 14, 17, 42 and 36 patients, respectively.

The relationship between tumour budding, clinicopathological characteristics, local host inflammatory response and TSP is presented in Table 1. Tumour budding was not significantly
A

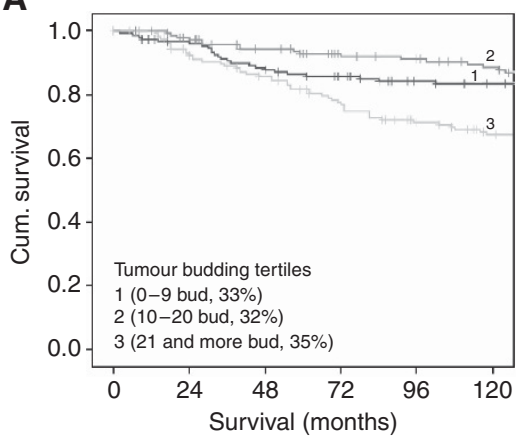

B

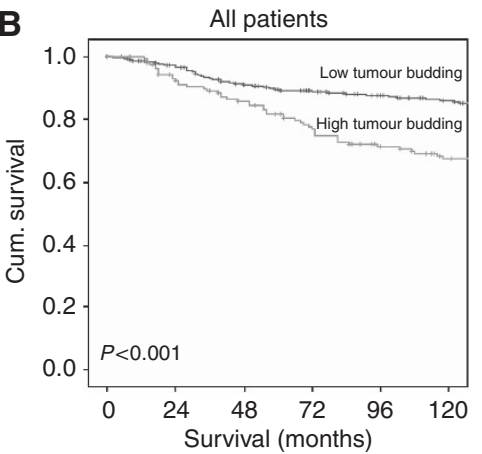

C

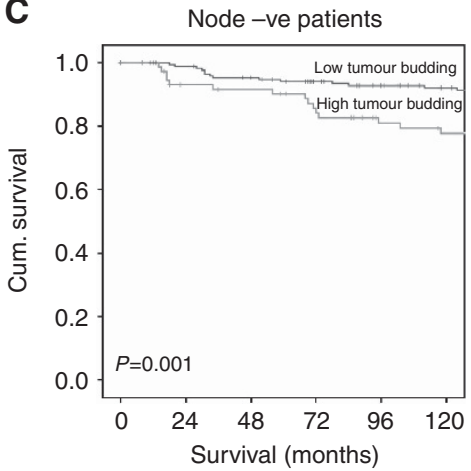

\begin{tabular}{r|cccccc}
$\begin{array}{r}\text { No. at } \\
\text { risk }\end{array} \begin{array}{r}\begin{array}{r}\text { Low } \\
\text { budding }\end{array} \\
\begin{array}{r}\text { High } \\
\text { budding }\end{array}\end{array}$ & 77 & 65 & 63 & 57 & 49 & 46
\end{tabular}
D

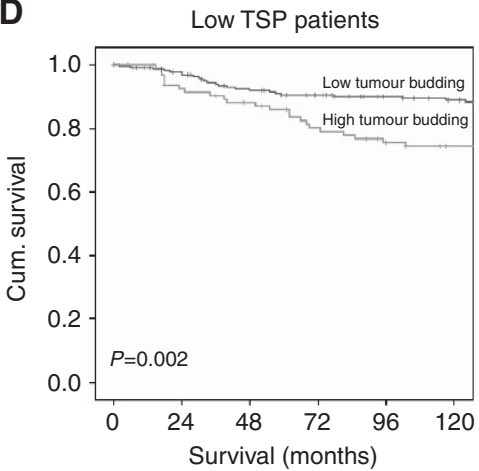

\begin{tabular}{lr|llllll}
$\begin{array}{c}\text { No. at } \\
\text { risk }\end{array}$ & $\begin{array}{r}\text { Low } \\
\text { budding }\end{array}$ & 214 & 199 & 183 & 172 & 164 & 160 \\
\hline $\begin{array}{r}\text { High } \\
\text { budding }\end{array}$ & 97 & 84 & 78 & 69 & 63 & 58
\end{tabular}
E

E High K-M score

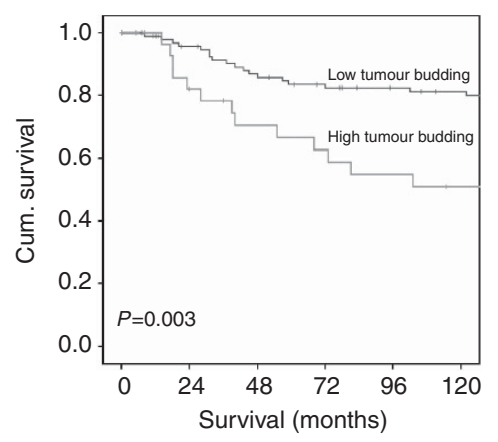

\begin{tabular}{lr|llllll}
$\begin{array}{c}\text { No. at } \\
\text { risk }\end{array}$ & $\begin{array}{r}\text { Low } \\
\text { budding }\end{array}$ & 93 & 83 & 74 & 69 & 65 & 62 \\
\hline $\begin{array}{r}\text { High } \\
\text { budding }\end{array}$ & 30 & 22 & 17 & 15 & 13 & 11
\end{tabular}

Figure 2. Kaplan-Meier survival curves (Log rank) of cancer specific survival (A) according to the tumour budding tertiles, (B) in all patients, (C) in patients with node negative tumours, (D) in patients with low TSP and (E) in patients with high K-M score. 
Table 1. Relationship between clinicopathological characteristics and tumour budding in patients with invasive ductal breast cancer (474)

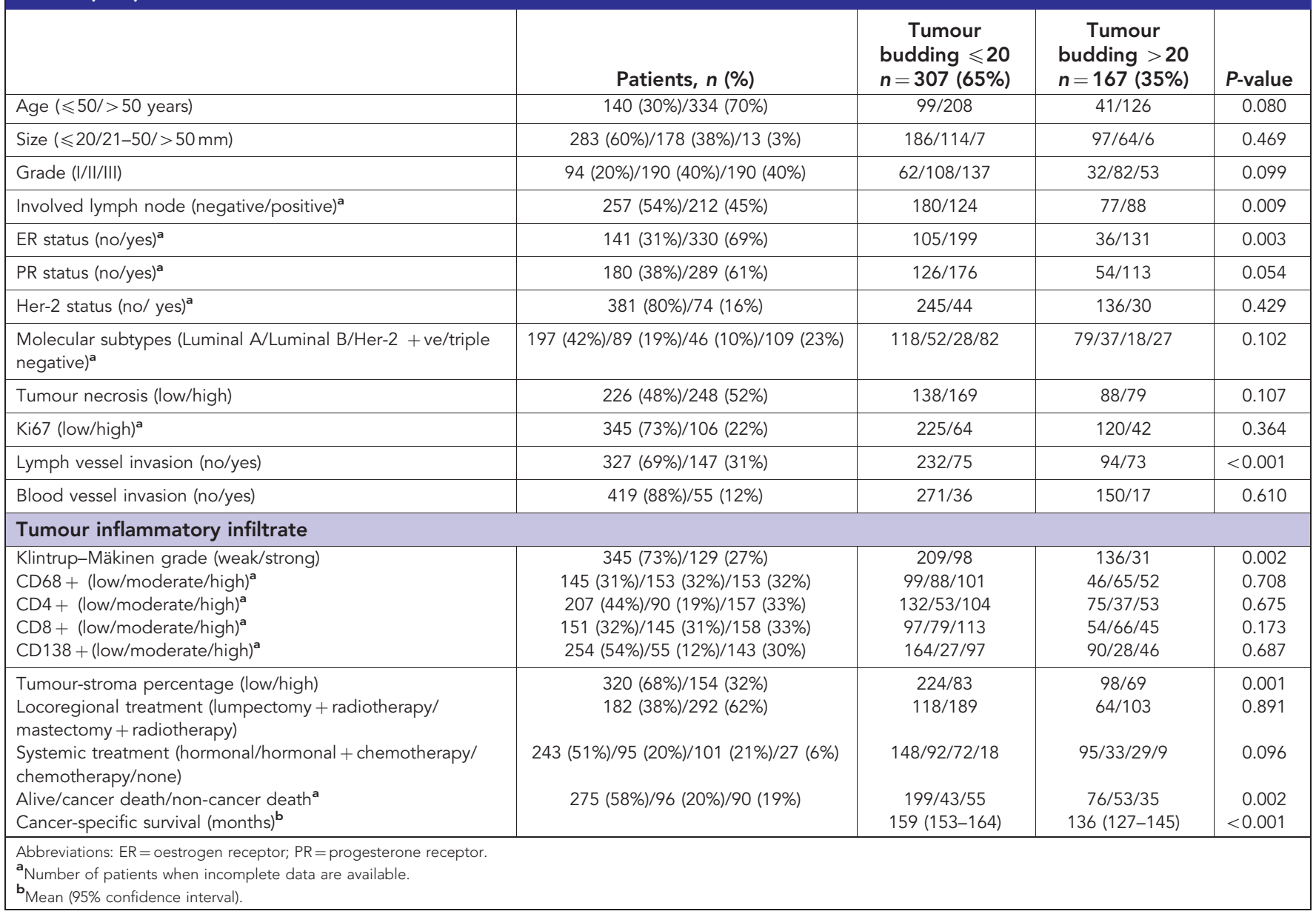

associated with age, size, grade, necrosis, Ki67 and BVI. A high tumour budding was associated with ER-positive status $(P=0.003)$, lymph node-positive tumours $(P=0.009)$, presence of LVI $(P<0.001)$ and high TSP $(P=0.001)$. A high tumour budding was inversely associated with local inflammatory response as measured by the $\mathrm{K}-\mathrm{M}$ score $(P=0.002)$ but not by macrophage, plasma cells and T-cell lymphocyte subtypes.

The relationship between clinicopathological characteristics and tumour budding in node-negative patients is presented in Table 2. A high tumour budding was associated with presence of LVI $(P<0.001)$ and inversely associated with local inflammatory response as measured by the $\mathrm{K}-\mathrm{M}$ score $(P=0.038)$. A high tumour budding showed a trend towards an association with TSP $(P=0.080)$.

The median survival of survivors was 164 months, with 96 deaths from breast cancer and 90 non-cancer deaths. In all, 13 (3\%) patients do not have survival data and were excluded from all survival analysis. Mean CSS was shorter in patients with high tumour budding compared with those with low tumour budding (136 vs 159 months, $P<0.001$; Figure 2B).

The relationship between tumour budding, clinicopathological characteristics and CSS is presented in Table 3. On univariate analysis, a high tumour budding was associated with shorter CSS $(P<0.001)$. On multivariate analysis, a high tumour budding was associated with reduced CSS (HR 1.96, 95\% CI 1.14-3.09, $P=0.004$ ), independent of nodal status, molecular subtypes, tumour necrosis, CD8 +, CD138 +, LVI, BVI and TSP.

In node-negative patients, a high tumour budding was associated with shorter mean CSS compared with a low tumour budding (150 vs 167 months, $P=0.001$; Figure $2 \mathrm{C}$ ). On multivariate survival analysis (Table 3), a high tumour budding was associated with reduced CSS
(HR 2.63, 95\% CI 1.16-5.92, $P=0.020$ ), independent of PR status, tumour necrosis, LVI, BVI and TSP.

In order to account for the high TSP and low cellular inflammatory infiltrate effects, sub-group survival analyses were performed based on low TSP and high K-M grade (Table 4). In stroma-low patients, a high tumour budding was associated with shorter mean CSS compared with a low tumour budding (144 vs 161 months, $P=0.002$; Figure $2 \mathrm{D}$ ). On multivariate survival analysis (Table 4), a high tumour budding was associated with reduced CSS (HR 1.98, 95\% CI 1.09-3.57, $P=0.024$ ), independent of molecular subtypes, tumour necrosis, LVI, BVI and CD68 + .

In patients with high $\mathrm{K}-\mathrm{M}$ score, a high tumour budding was associated with shorter mean CSS compared with a low tumour budding (110 vs 151 months, $P=0.003$; Figure 2D). On multivariate survival analysis (Table 4), a high tumour budding was associated with reduced CSS (HR 2.27, 95\% CI 1.35-5.36, $P=0.014$ ), independent of molecular subtypes, LVI, BVI, CD68 +, CD8 + and TSP.

When survival analysis for tumour budding was performed across the different molecular subtypes, a high tumour budding was associated with shorter mean CSS compared with a low tumour budding in Luminal B patients (121 vs 152 months, $P=0.008$ ), Her-2 patients (89 vs 142 months, $P=0.019$ ) and triple-negative patients (110 vs 144 months, $P=0.020)$ (Figure 3).

\section{DISCUSSION}

In the present study, high tumour budding was associated with more tumour stroma and a weaker inflammatory cell infiltrate and was independently associated with reduced CSS. These results 
Table 2. Relationship between clinicopathological characteristics and tumour budding in patients with node-negative invasive ductal breast cancer $(n=257)$

\begin{tabular}{|c|c|c|c|}
\hline & $\begin{array}{c}\text { Tumour budding } \leqslant 20 \\
n=180(70 \%)\end{array}$ & $\begin{array}{c}\text { Tumour budding }>20 \\
n=77(30 \%)\end{array}$ & $P$-value \\
\hline Age $(\leqslant 50 />50$ years $)$ & $49 / 131$ & $23 / 54$ & 0.666 \\
\hline Size $(\leqslant 20 / 21-50 />50 \mathrm{~mm})$ & $125 / 54 / 1$ & $56 / 20 / 1$ & 0.696 \\
\hline Grade (I/II/III) & $45 / 62 / 73$ & $18 / 41 / 18$ & 0.137 \\
\hline ER status (no/yes) & $57 / 122$ & $18 / 59$ & 0.173 \\
\hline PR status (no/yes) & $74 / 103$ & $23 / 54$ & 0.072 \\
\hline Her-2 status (no/yes) & $151 / 21$ & $66 / 10$ & 0.835 \\
\hline Molecular subtypes (Luminal A/Luminal B/Her-2 + ve/triple negative) & $77 / 27 / 13 / 50$ & $43 / 13 / 6 / 11$ & 0.065 \\
\hline Tumour necrosis (low/high) & 93/87 & $49 / 28$ & 0.078 \\
\hline Ki67 (low/high) & $136 / 34$ & $62 / 12$ & 0.488 \\
\hline Lymph vessel invasion (no/yes) & $153 / 27$ & $50 / 27$ & $<0.001$ \\
\hline Blood vessel invasion (no/yes) & $162 / 18$ & $69 / 8$ & 0.925 \\
\hline \multicolumn{4}{|l|}{ Tumour inflammatory infiltrate } \\
\hline $\begin{array}{l}\text { Klintrup-Mäkinen grade (weak/strong) } \\
\text { CD68 + (low/moderate/high) } \\
\text { CD4 + (low/moderate/high) } \\
\text { CD8 + (low/moderate/high) } \\
\text { CD138 + (low/moderate/high) } \\
\text { Tumour-stroma percentage (low/high) } \\
\text { Locoregional treatment (lumpectomy + radiotherapy/mastectomy + radiotherapy) } \\
\text { Systemic treatment (hormonal/hormonal + chemotherapy/chemotherapy/none } \\
\text { Alive/cancer death/non-cancer death } \\
\text { Cancer-specific survival (months) }\end{array}$ & $\begin{array}{c}133 / 47 \\
65 / 52 / 52 \\
80 / 31 / 59 \\
60 / 46 / 64 \\
102 / 15 / 52 \\
224 / 83 \\
84 / 96 \\
105 / 20 / 36 / 17 \\
126 / 15 / 33 \\
167(162-173)\end{array}$ & $\begin{array}{c}66 / 11 \\
26 / 26 / 23 \\
38 / 14 / 24 \\
26 / 31 / 19 \\
45 / 15 / 15 \\
98 / 69 \\
37 / 40 \\
54 / 6 / 8 / 8 \\
45 / 17 / 15 \\
150(138-168)\end{array}$ & $\begin{array}{l}0.038 \\
0.747 \\
0.623 \\
0.313 \\
0.393 \\
0.080 \\
0.832 \\
0.142 \\
0.184 \\
0.001\end{array}$ \\
\hline
\end{tabular}

suggest a complex relationship between tumour budding and the tumour microenvironment and disease progression in patients with invasive ductal breast cancer.

Few studies have examined the prognostic value of tumour budding in breast cancer (Liang et al, 2013; Salhia et al, 2015). However, the prognostic value and method of assessment of tumour budding in colorectal cancer has recently been reviewed by van Wyk et al (2015). They concluded that IHC did not improve the detection rate or the prognostic value of tumour budding over that of H\&E (van Wyk et al, 2015).Therefore, in the present study, the $H \& E$ approach was used.

In the present study, examination of tumour budding was by semiquantitative method and was reproducible $(\mathrm{ICCC}=0.813$ ). Patients in the present study were divided into three budding groups based on tertiles. The cutoff considered the best discriminator of CSS (Choi et al, 2007; Sy et al, 2010) was between groups 2 and 3 and yielded a cutoff consistent with previous reports (16-25 buds) (Prall et al, 2005; Wang et al, 2009). Furthermore, in the present study tumour budding was found in $35 \%$ of patients and is consistent with previous report in patients with breast cancer (Liang et al, 2013).

The results of the present study showed that high grade budding was significantly associated with ER-positive tumours. These results are consistent with the recent observations of Salhia et al (2015) using a pan-cytokeratin stain to assess tumour budding. The basis of these observations is not clear. However, it was recently reported that oestrogen is involved in EMT in breast cancer cell lines with stem cell properties (Sun et al, 2014) and that oestrogen is involved in disruption of tight junction and increased cell motility (Sanchez et al, 2010; Jiménez-Salazar et al, 2014). Therefore, this may suggest that ER-positive tumours with high tumour budding may be undergoing a higher degree of EMT and as a result more metastatic potential. If this were to be the case, then it might be expected that anti-oestrogen treatment would reduce the degree of budding in those patients.
Despite being associated with lymph node metastasis and lymph vessel invasion, tumour budding was not associated with blood vessel invasion. The basis of this observation was not clear; however, tumour buds may find their way of metastasis through invasion into lymph vessels than blood vessels, as it is the major route of metastasis in breast cancer (Mohammed et al, 2009; Gujam et al, 2014b). In the present study, there was a lack of any perceived association between tumour budding and tumour size, grade, necrosis or Ki67 in all cohort and in sub-group analysis. Previous breast and colorectal cancers studies reported that budded cells displayed lower proliferation activity rather than high proliferative activity (Palmqvist et al, 2000; Liang et al, 2013; Dawson and Lugli, 2015). This may suggest that detachment and dissociation of tumour cells are not influenced by increased tumour size, its differentiation or proliferation activities.

Although the inter-relationships between the tumour budding, tumour microenvironment and gross pathological characteristics are likely complex, tumour budding remained independently associated with CSS in different patient sub-groups. In high-risk patients with node-negative disease, tumour budding was significantly associated with reduced CSS alongside with tumour necrosis, LVI and BVI. Indeed, the present results further confirm the importance of both tumour and host-based factors of the tumour microenvironment in determining cancer outcome.

Although there is now increased appreciation of the importance of the tumour budding in cancer progression and survival in several previous reports (Hase et al, 1993; Ueno et al, 2002; Prall et al, 2005; Koike et al, 2008; Masugi et al, 2010; Koyuncuoglu et al, 2012; Taira et al, 2012; Liang et al, 2013), its relationship with other components of the tumour microenvironment has yet to be fully characterised. It was of interest that the present study found an association between tumour budding and increased amount of TSP. Earlier reports in colorectal cancer have shown an association between tumour budding and the presence of an immature stroma and a high density of stromal myofibroblasts (Ueno et al, 2004). 
Table 3. Relationship between clinicopathological characteristics and cancer-specific survival in patients with invasive ductal breast cancer

\begin{tabular}{|c|c|c|c|c|}
\hline & \multirow{2}{*}{\multicolumn{2}{|c|}{ Univariate analysis }} & \multirow{2}{*}{\multicolumn{2}{|c|}{ Multivariate analysis }} \\
\hline & & & & \\
\hline All patients $(n=461)$ & $\begin{array}{c}\text { Hazard ratio } \\
(95 \% \mathrm{Cl})\end{array}$ & $P$-value & $\begin{array}{c}\text { Hazard ratio } \\
(95 \% \mathrm{Cl})\end{array}$ & $P$-value \\
\hline Age $(\leqslant 50 />50$ years $)$ & $1.22(0.77-1.91)$ & 0.397 & & \\
\hline Size $(\leqslant 20 / 21-50 />50 \mathrm{~mm})$ & $2.11(1.49-2.97)$ & $<0.001$ & & 0.717 \\
\hline Grade (I/II/IIII) & $1.87(1.38-2.53)$ & $<0.001$ & & 0.491 \\
\hline Involved lymph node (no/yes) & $2.76(1.80-4.23)$ & $<0.001$ & $1.54(0.95-2.45)$ & 0.081 \\
\hline ER status (no/yes) & $0.617(0.41-0.93)$ & 0.021 & & 0.905 \\
\hline PR status (no/yes) & $0.54(0.36-0.81)$ & 0.003 & & 0.646 \\
\hline Her-2 status (no/yes) & $2.02(1.27-3.22)$ & 0.003 & & 0.216 \\
\hline Molecular subtypes (Luminal A/Luminal B/Her-2 + ve/triple negative) & $1.61(1.34-1.94)$ & $<0.001$ & $1.50(1.22-1.84)$ & $<0.001$ \\
\hline Tumour necrosis (low/high) & $1.97(1.48-8.59)$ & 0.005 & $2.53(1.41-4.53)$ & 0.002 \\
\hline Lymph vessel invasion (no/yes) & $4.14(2.75-6.29)$ & $<0.001$ & $2.09(1.28-3.40)$ & 0.003 \\
\hline Blood vessel invasion (no/yes) & $3.39(2.14-5.39)$ & $<0.001$ & $2.23(1.35-3.69)$ & 0.002 \\
\hline Klintrup-Mäkinen grade (weak/strong) & $1.48(0.96-2.26)$ & 0.069 & & 0.488 \\
\hline CD68 + (low/moderate/high) & $0.86(0.67-1.09)$ & 0.222 & & \\
\hline CD4 + (low/moderate/high) & $1.00(0.80-1.25)$ & 0.983 & & \\
\hline CD8 + (low/moderate/high) & $0.69(0.54-0.88)$ & 0.004 & $0.54(0.41-0.71)$ & $<0.001$ \\
\hline CD138 + (low/moderate/high) & $1.38(1.11-1.71)$ & 0.003 & $1.01(1.01-1.02)$ & 0.002 \\
\hline Tumour stroma percentage (low/high) & $2.19(1.46-3.27)$ & $<0.001$ & $1.74(1.14-2.66)$ & 0.010 \\
\hline Tumour budding (low/high) & $2.53(1.69-3.78)$ & $<0.001$ & $1.96(1.14-3.09)$ & 0.004 \\
\hline \multicolumn{5}{|l|}{ Node-negative patients $(n=251)$} \\
\hline Age $(\leqslant 50 />50$ years $)$ & $1.22(0.55-2.71)$ & 0.632 & & \\
\hline Size $(\leqslant 20 / 21-50 />50 \mathrm{~mm})$ & $2.49(1.25-4.97)$ & 0.010 & & 0.323 \\
\hline Grade (I/II/III) & $1.67(1.03-2.72)$ & 0.038 & & 0.884 \\
\hline ER status (no/yes) & $0.48(0.24-0.97)$ & 0.040 & & 0.693 \\
\hline PR status (no/yes) & $0.39(0.19-0.81)$ & 0.010 & $0.41(0.19-0.87)$ & 0.020 \\
\hline Her-2 status (no/yes) & $1.75(0.72-4.26)$ & 0.221 & & \\
\hline Molecular subtypes (Luminal A/Luminal B/Her-2 + ve/triple negative) & $1.57(1.12-2.18)$ & 0.008 & & 0.276 \\
\hline Tumour necrosis (low/high) & $3.75(1.73-8.11)$ & 0.001 & $3.02(1.31-6.92)$ & 0.009 \\
\hline Lymph vessel invasion (no/yes) & $4.67(2.33-9.36)$ & $<0.001$ & $3.11(1.39-6.97)$ & 0.006 \\
\hline Blood vessel invasion (no/yes) & $3.95(1.77-8.80)$ & 0.001 & $2.66(1.09-6.45)$ & 0.030 \\
\hline Klintrup-Mäkinen grade (weak/strong) & $1.45(0.67-3.13)$ & 0.347 & & \\
\hline CD68 + (low/moderate/high) & $0.52(0.39-1.35)$ & 0.643 & & \\
\hline CD4 + (low/moderate/high) & $1.04(0.63-1.21)$ & 0.872 & & \\
\hline CD8 + (low/moderate/high) & $0.653(0.324-1.15)$ & 0.132 & & \\
\hline CD138 + (low/moderate/high) & $1.13(0.48-1.63)$ & 0.625 & & \\
\hline Tumour stroma percentage (low/high) & $1.46(1.84-3.66)$ & 0.014 & & 0.087 \\
\hline Tumour budding (low/high) & $2.83(1.46-5.86)$ & 0.003 & $2.63(1.16-5.92)$ & 0.020 \\
\hline
\end{tabular}

Furthermore, tumour stroma have been implicated to facilitate EMT, one of the features of budded cells (Masugi et al, 2010; Zlobec and Lugli, 2010; Koyuncuoglu et al, 2012; Lugli et al, 2012; Taira et al, 2012; Liang et al, 2013), and metastasis of tumour cells into normal tissue (De Wever and Mareel, 2003; Hemmings 2013). Therefore, the present finding may support an important role of the tumour stroma in facilitating tumour cell de-differentiation and dissemination, perhaps providing suitable energy substrate and reducing the build-up of metabolic waste (Koukourakis et al, 2006).

Of interest, the present study has characterised the relationship between tumour budding and local host inflammatory infiltrate. There was a weaker peritumoural inflammatory infiltrate, as measured by $\mathrm{K}-\mathrm{M}$ score but not by individual subtypes of innate or adaptive immune cells, in patients with high-grade tumour budding. This may suggest that tumour budding may promote the development of a pro-tumour rather than antitumour immune response. It is of interest that the prognostic value of the ratio of CD8 and budding has recently been reported in primary operable colorectal cancer and showed that a high tumour budding and a low CD8 + T-lymphocyte index was associated with tumour progression and worse survival (Lugli et al, 2009), confirming the pro-tumour impact of the tumour budding. However, when we examined CD8/budding index in the present breast cancer cohort, the CD8/budding index did not show additional prognostic value to that of tumour budding alone. Therefore, further work is required to establish the prognostic value of the CD8/budding index in patients with cancer.

Given that tumour budding has independent prognostic value in patients with primary operable ductal breast cancer, it would be of interest to examine the prognostic value of intra-tumoural budding (ITB), as if this was the case then it may be applied to the initial diagnostic biopsy samples to better predict likely outcome and plan treatment prior to surgery. For example, if ITB was strongly associated with lymph node metastases, then it may be that the corresponding sentinel lymph nodes should be analysed carefully on frozen sections in preoperative biopsies. Indeed, Zlobec et al (2014) reported that ITB in preoperative biopsies predicts the presence of lymph node and distant metastases in colorectal cancer patients. However, Salhia et al (2015) reported that, in breast cancer, ITB in preoperative core biopsies was associated with blood 
Table 4. Relationship between clinicopathological characteristics and cancer-specific survival in patients with low TSP and high $\mathrm{K}-\mathrm{M}$ score

\begin{tabular}{|c|c|c|c|c|}
\hline \multirow[b]{2}{*}{ Stroma-low patients $(n=311)$} & \multicolumn{2}{|c|}{ Univariate analysis } & \multicolumn{2}{|c|}{ Multivariate analysis } \\
\hline & Hazard ratio $(95 \% \mathrm{Cl})$ & $P$-value & Hazard ratio $(95 \% \mathrm{Cl})$ & $P$-value \\
\hline Age $(\leqslant 50 />50$ years $)$ & $1.04(0.59-1.86)$ & 0.885 & & \\
\hline Size $(\leqslant 20 / 21-50 />50 \mathrm{~mm})$ & $2.73(1.69-4.40)$ & $<0.001$ & & 0.224 \\
\hline Grade (I/II/III) & $1.93(1.27-2.94)$ & 0.002 & & 0.956 \\
\hline Involved lymph node (no/yes) & $2.52(1.45-4.39)$ & 0.001 & & 0.302 \\
\hline ER status (no/yes) & $0.52(0.30-0.92)$ & 0.023 & & 0.814 \\
\hline PR status (no/yes) & $0.65(0.32-1.06)$ & 0.072 & & 0.437 \\
\hline Her-2 status (no/yes) & $1.96(1.04-3.70)$ & 0.036 & & 0.960 \\
\hline Molecular subtypes (Luminal A/Luminal B/ Her-2 + ve/triple negative) & $1.66(1.28-2.16)$ & $<0.001$ & $1.58(1.18-2.13)$ & 0.002 \\
\hline Tumour necrosis (low/high) & $4.59(2.30-9.14)$ & $<0.001$ & $2.89(1.35-6.27)$ & 0.007 \\
\hline Lymph vessel invasion (no/yes) & $4.59(2.63-8.02)$ & $<0.001$ & $2.76(1.49-5.12)$ & 0.003 \\
\hline Blood vessel invasion (no/yes) & $5.49(3.13-9.64)$ & $<0.001$ & $4.43(2.38-8.25)$ & $<0.001$ \\
\hline Klintrup-Mäkinen grade (weak/strong) & $1.64(0.93-2.08)$ & 0.076 & & 0.105 \\
\hline CD68 + (low/moderate/high) & $0.99(0.99-1.00)$ & 0.028 & $0.95(0.92-0.99)$ & 0.003 \\
\hline CD4 + (low/moderate/high) & $0.01(0.09-1.01)$ & 0.337 & & \\
\hline CD8 + (low/moderate/high) & $0.99(0.99-1.02)$ & 0.243 & & \\
\hline CD138 + (low/moderate/high) & $1.01(0.99-1.01)$ & 0.092 & & 0.261 \\
\hline Tumour budding (low/high) & $2.29(1.32-3.95)$ & 0.002 & $1.98(1.09-3.57)$ & 0.024 \\
\hline \multicolumn{5}{|l|}{ High Klintrup-Mäkinen grade patients $(n=123)$} \\
\hline Age $(\leqslant 50 />50$ years $)$ & $0.73(0.36-1.45)$ & 0.365 & & \\
\hline Size $(\leqslant 20 / 21-50 />50 \mathrm{~mm})$ & $1.86(0.93-3.74)$ & 0.081 & & 0.735 \\
\hline Grade $(I / I / I I I)$ & $1.75(0.71-4.32)$ & 0.226 & & \\
\hline Involved lymph node (no/yes) & $2.35(1.09-5.09)$ & 0.030 & & 0.836 \\
\hline ER status (no/yes) & $0.60(0.29-1.23)$ & 0.165 & & \\
\hline PR status (no/yes) & $0.49(0.22-1.10)$ & 0.496 & & \\
\hline Her-2 status (no/ yes) & $1.22(0.59-2.53)$ & 0.598 & & \\
\hline Molecular subtypes (Luminal A/Luminal B/Her-2 + ve/triple negative) & $1.55(1.05-2.30)$ & 0.029 & $2.49(1.50-4.12)$ & $<0.001$ \\
\hline Tumour necrosis (low/high) & $23.78(0.16-34.34)$ & 0.213 & & \\
\hline Lymph vessel invasion (no/yes) & $6.25(2.65-14.49)$ & $<0.001$ & $5.84(2.39-14.25)$ & $<0.001$ \\
\hline Blood vessel invasion (no/yes) & $3.94(1.89-8.20)$ & $<0.001$ & $4.15(1.76-9.74)$ & 0.001 \\
\hline CD68 + (low/moderate/high) & $0.95(0.92-0.99)$ & 0.004 & $0.95(0.94-0.99)$ & 0.027 \\
\hline CD4 + (low/moderate/high) & $0.99(0.99-1.01)$ & 0.300 & & \\
\hline CD8 + (low/moderate/high) & $0.99(0.98-0.99)$ & 0.006 & $0.99(0.98-1.00)$ & 0.092 \\
\hline CD138 + (low/moderate/high) & $1.01(0.99-1.01)$ & 0.164 & & \\
\hline Tumour stroma percentage (low/high) & $2.62(1.26-5.45)$ & 0.010 & $2.41(1.09-5.30)$ & 0.030 \\
\hline Tumour budding (low/high) & $2.82(1.39-5.72)$ & 0.003 & $2.27(1.35-5.36)$ & 0.041 \\
\hline
\end{tabular}

vessel invasion but not with lymphatic and nodal invasion. Nevertheless, prospective studies comparing the prognostic value of tumour budding in preoperative core biopsies and resection specimens would be of great interest.

Taken together, the present results suggest that tumour budding may promote disease progression through a direct effect on local and distant invasion into lymph nodes and lymphatic vessels. Indeed, budded cells have been shown to display epithelialmesenchymal transition-like molecular phenotype in several cancers (Masugi et al, 2010; Zlobec and Lugli, 2010; Koyuncuoglu et al, 2012, Lugli et al, 2012; Taira et al, 2012; Liang et al, 2013), which is an early and critical step in cancer metastasis (Kalluri and Weinberg, 2009). Tumour with low inflammatory infiltrate may become more aggressive and allow tumour cells to detach and invade locally and systematically. Indeed, results from the present study and from our previous work (Gujam et al, 2014a) also suggest that both tumour budding and tumour stroma support pro-tumour rather than antitumour immune response.

The results of the present study suggest that tumour budding should be incorporated into routine clinical practice. However, in order for that to occur it has to be shown to be a reliable measure.
Although several studies have confirmed the prognostic value of tumour budding, several different methods have been described (Hase et al, 1993; Ueno et al, 2002; Prall et al, 2005; Wang et al, 2009). Therefore, there is a need for a standardised method to assess tumour budding in patients with cancer. In particular, if the standardised assessment of the tumour budding can reliably be performed in routine pathological sections and can offer useful prognostic information for clinicians, this would form the platform for the integration of tumour budding into existing staging systems.

With reference to patients with breast cancer, to date, tumour budding has been rarely examined, and therefore, the results of the present study need to be externally validated. Furthermore, whether tumour budding could be used as an additional morphological feature to stratify ER-positive into a high- and low-risk category has also to be validated.

\section{CONCLUSION}

In summary, the present study provides comprehensive assessment of the associations between tumour budding and the tumour microenvironment and, in a mature cohort of patients with long- 
A
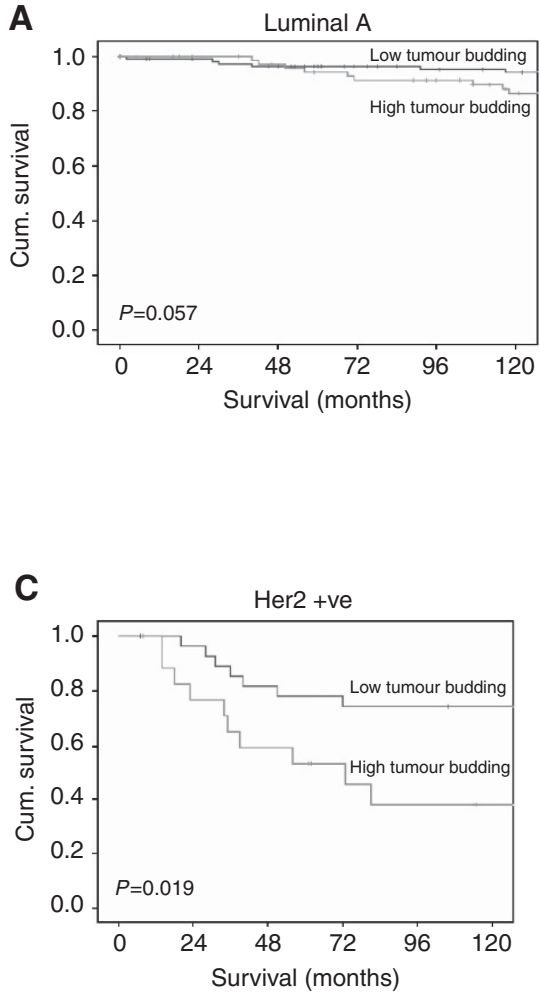

B
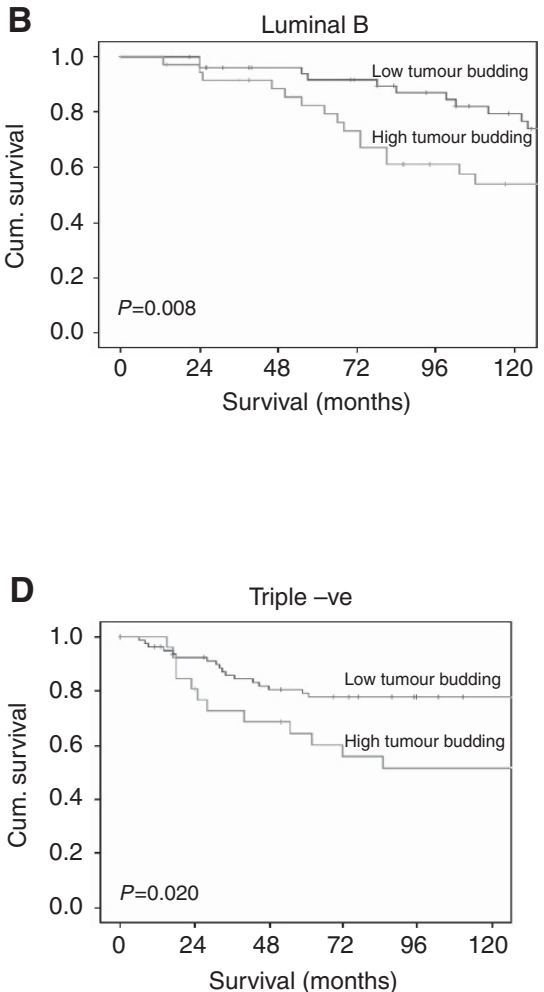

Figure 3. Kaplan-Meier survival curves (Log rank) of cancer specific survival in different molecular subtypes (A) patients with Luminal A, (B) patients with Luminal B, (C) patients with Her-2 positive tumours and (D) patients with Triple negative tumours.

term follow-up, further confirms the prognostic relevance of assessment of the tumour microenvironment in patients with invasive ductal breast cancer. Assessment of the tumour budding utilising routine pathological slides is relatively simple and may be readily incorporated into routine clinical pathology reporting to improve risk stratification, in particular for patients with nodenegative breast cancer.

\section{ACKNOWLEDGEMENTS}

We gratefully acknowledge the role of Clare Orange for her technical assistance in Slidepath.

\section{REFERENCES}

Cancerstats (2014) 17-01-2015. Ref. type: Online Source http://www.cancerresearchuk.org/cancer-info/cancerstats/types/breast/.

Choi HJ, Park KJ, Shin JS, Roh MS, Kwon HC, Lee HS (2007) Tumor budding as a prognostic marker in stage-III rectal carcinoma. Int J Colorectal Dis 22: 863-868.

Dawson H, Lugli A (2015) Molecular and pathogenetic aspects of tumor budding in colorectal cancer. Front Med 2: 11.

De Wever O, Mareel M (2003) Role of tissue stroma in cancer cell invasion. $J$ Pathol 200: 429-447.

Freeman MR, Li Q, Chung LWK (2013) Can stroma reaction predict cancer lethality? Clin Cancer Res 19: 4905-4907.

Gujam FJA, Edwards J, Mohammed ZMA, Going JJ, McMillan DC (2014a) The relationship between the tumour stroma percentage, clinicopathological characteristics and outcome in patients with operable ductal breast cancer. Br J Cancer 111: 157-165.

Gujam FJA, Going JJ, Mohammed ZMA, Orange C, Edwards J, McMillan DC (2014b) Immunohistochemical detection improves the prognostic value of lymphatic and blood vessel invasion in primary ductal breast cancer. BMC Cancer 14: 676.
Hammond ME, Hayes DF, Dowsett M, Allred DC, Hagerty KL, Badve S, Fitzgibbons PL, Francis G, Goldstein NS, Hayes M, Hicks DG, Lester S, Love R, Mangu PB, McShane L, Miller K, Osborne CK, Paik S, Perlmutter J, Rhodes A, Sasano H, Schwartz JN, Sweep FCG, Taube S, Torlakovic EE, Valenstein P, Viale G, Visscher D, Wheeler T, Williams RB, Wittliff JL, Wolff AC. American Society of Clinical OncologyCollege of American Pathologists (2010) American society of clinical oncology/college of American pathologists guideline recommendations for immunohistochemical testing of estrogen and progesterone receptors in breast cancer. J Clin Oncol 28: 2784-2795.

Hase K, Shatney C, Johnson D, Trollope M, Vierra M. Prognostic value of tumor budding in patients with colorectal-cancer (1993) Dis Colon Rectum 36: $627-635$.

Hemmings C (2013) Is carcinoma a mesenchymal disease? The role of the stromal microenvironment in carcinogenesis. Pathology 45: 371-381.

Jiménez-Salazar JE, Posadas-Rodriquez P, Lazzarini-Lechuga RC, Luna-Lopez A, Zentella-Dehesa A, Gomez-Quiroz LE, Königsberg M, Dominquez-Gomez G, Damian-Matsumura P (2014) Membrane-initiated estradiol signaling of epithelial-mesenchymal transition-associated mechanisms through regulation of tight junctions in human breast cancer cells. Horm Cancer 5: 161-173.

Kalluri R, Weinberg RA (2009) The basics of epithelial-mesenchymal transition. J Clin Invest 119: 1420-1428.

Klintrup K, Makinen JM, Kauppila S, Vare PO, Melkko J, Tuominen H, Tuppurainen K, Makela J, Karttunen TJ, Makinen MJ (2005) Inflammation and prognosis in colorectal cancer. Eur J Cancer 41: 2645-2654.

Koike M, Kodera Y, Itoh Y, Nakayama G, Fujiwara M, Hamajima N, Nakao A (2008) Multivariate analysis of the pathologic features of esophageal squamous cell cancer: tumor budding is a significant independent prognostic factor. Ann Surg Oncol 15: 1977-1982.

Koukourakis MI, Giatromanolaki A, Harris AL, Sivridis E (2006) Comparison of metabolic pathways between cancer cells and stromal cells in colorectal carcinomas: a metabolic survival role for tumor-associated stroma. Cancer Res 66: 632-637.

Koyuncuoglu M, Okyay E, Saatli B, Olgan S, Akin M, Saygili U (2012) Tumor budding and E-Cadherin expression in endometrial carcinoma: 
are they prognostic factors in endometrial cancer? Gynecol Oncol 125: 208-213.

Liang FL, Cao W, Wang YL, Li LR, Zhang GJ, Wang Z (2013) The prognostic value of tumor budding in invasive breast cancer. Pathol Res Pract 209: 269-275.

Lugli A, Karamitopoulou E, Panayiotides I, Karakitsos P, Rallis G, Peros G, Iezzi G, Spagnoli G, Bihll M, Terracciano L, Zlobec I (2009) CD8 + lymphocytes/tumour-budding index: an independent prognostic factor representing a 'pro-/anti-tumour' approach to tumour host interaction in colorectal cancer. Br J Cancer 101: 1382-1392.

Lugli A, Karamitopoulou E, Zlobec I (2012) Tumour budding: a promising parameter in colorectal cancer. BrJ Cancer 106: 1713-1717.

Masugi Y, Yamazaki K, Hibi T, Kitagawa Y, Sakamoto M (2010) Solitary cell infiltration is a novel indicator of poor prognosis and epithelialmesenchymal transition in pancreatic cancer. Hum Pathol 41: 1061-1068.

Mohammed RA, Ellis I, Lee A, Martin S (2009) Vascular invasion in breast cancer; an overview of recent prognostic developments and molecular pathophysiological mechanisms. Histopathology 55: 1-9.

Mohammed ZMA, Going JJ, Edwards J, Elsberger B, Doughty JC, McMillan DC (2012a) The relationship between components of tumour inflammatory cell infiltrate and clinicopathological factors and survival in patients with primary operable invasive ductal breast cancer. $\mathrm{Br} J$ Cancer 107: 864-873.

Mohammed ZMA, Going JJ, Edwards J, Elsberger B, McMillan DC (2013) The relationship between lymphocyte subsets and clinico-pathological determinants of survival in patients with primary operable invasive ductal breast cancer. Br J Cancer 109: 1676-1684.

Mohammed ZMA, Going JJ, McMillan DC, Orange C, Mallon E, Doughty JC, Edwards J (2012b) Comparison of visual and automated assessment of HER2 status and their impact on outcome in primary operable invasive ductal breast cancer. Histopathology 61: 675-684.

Palmqvist R, Rutegard JN, Bozoky B, Landberg G, Stenling R (2000) Human colorectal cancers with an intact p16/cyclin D1/pRb pathway have upregulated p16 expression and decreased proliferation in small invasive tumor clusters. Am J Pathol 157: 1947-1953.

Prall F, Nizze H, Barten M (2005) Tumour budding as prognostic factor in stage I/II colorectal carcinoma. Histopathology 47: 17-24.

Richards CH, Mohammed Z, Qayyum T, Horgan PG, McMillan DC (2011) The prognostic value of histological tumor necrosis in solid organ malignant disease: a systematic review. Future Oncol 7: 1223-1235.

Salhia B, Trippel M, Pfaltz K, Cihoric N, Grogg A, Lädrach C, Zlobec I, Tapia C (2015) High tumour budding stratify breast cancer with metastatic properties. Breast Cancer Res Treat 150: 363-371.
Sanchez AM, Flamini MI, Baldacci C, Goglia L, Genazzani AR, Simoncini T (2010) Estrogen receptor- $\alpha$ promotes breast cancer cell motility and invasion via focal adhesion kinase and N-WASP. Mol Endocrinol 24: 2114-2125.

Sun Y, Wang Y, Fan C, Gao P, Wang X, Wie G, Wie J (2014) Estrogen promotes stemness and invasiveness of ER-positive breast cancer cells through Glil activation. Mol Cancer 13: 137.

Sy J, Fung CLS, Dent OF, Chapuis PH, Bokey L, Chan C (2010) Tumor budding and survival after potentially curative resection of node-positive colon cancer. Dis Colon Rectum 53: 301-307.

Taira T, Ishii G, Nagai K, Yoh K, Takahashi Y, Matsumura Y, Kojima M, Ohmatsu H, Goto K, Niho S, Takashima H, Inoue H, Ohe Y, Ochiai A (2012) Characterization of the immunophenotype of the tumor budding and its prognostic implications in squamous cell carcinoma of the lung. Lung Cancer 76: 423-430.

Ueno H, Jones AM, Wilkinson KH, Jass JR, Talbot IC (2004) Histological categorisation of fibrotic cancer stroma in advanced rectal cancer. Gut 53: 581-586.

Ueno H, Murphy J, Jass JR, Mochizuki H, Talbot IC (2002) Tumour 'budding' as an index to estimate the potential of aggressiveness in rectal cancer. Histopathology 40: 127-132.

van Wyk HC, James P, Roxburgh C, Horgan P, Foulis A, McMillan DC (2015) The role of tumour budding in predicting survival in patients with primary operable colorectal cancer: a systematic review. Cancer Treat Rev 41: 151-159.

Wang LM, Kevans D, Mulcahy H, O'Sullivan J, Fennelly D, Hyland J, Diarmuid O, Sheahan K (2009) Tumor budding is a strong and reproducible prognostic marker in T3N0 colorectal cancer. Am J Surg Pathol 33: 134-141.

Zlobec I, Lugli A (2010) Epithelial mesenchymal transition and tumor budding in aggressive colorectal cancer: tumor budding as oncotarget. Oncotarget 1: 651-661.

Zlobec I, Hädrich M, Dawson H, Koelzer VH, Borner M, Mallaev M, Schnüriger B, Inderbitzin D, Lugli A (2014) Intratumoral budding (ITB) in preoperative biopsies predicts the presence of lymph node and distant metastases in colon and rectal cancer patients. Br J Cancer 110: 1008-1013.

This work is published under the standard license to publish agreement. After 12 months the work will become freely available and the license terms will switch to a Creative Commons AttributionNonCommercial-Share Alike 4.0 Unported License. 